\title{
An Infrastructure for Developing Pervasive Learning Environments
}

\author{
Sabine Graf ${ }^{1}$, Kathryn MacCallum ${ }^{2}$, Tzu-Chien $\mathrm{Liu}^{3}$, Maiga Chang ${ }^{4}$, Dunwei Wen ${ }^{4}$, Qing Tan $^{4}$, \\ Jon Dron ${ }^{4}$, Fuhua Lin ${ }^{4}$, Nian-Shing Chen ${ }^{5}$, Rory McGreal ${ }^{4}$, Kinshuk ${ }^{4}$ \\ ${ }^{1}$ Vienna University of Technology, Austria, sabine.graf@ieee.org \\ ${ }^{2}$ Massey University, New Zealand, k.maccallum@massey.ac.nz \\ ${ }^{3}$ National Central University, Taiwan,ltc@cc.ncu.edu.tw \\ ${ }^{4}$ Athabasca University, Canada \\ \{maigac|dunweiw|qingt|jond|oscarl|rory|kinshuk@athabascau.ca\} \\ ${ }^{5}$ National Sun Yat-sen University, Taiwan,nschen@cc.nsysu.edu.tw
}

\begin{abstract}
This paper presents an infrastructure for developing problem-based pervasive learning environments. Building such environments necessitates having many autonomous components dealing with various tasks and heterogeneous distributed resources. Our proposed infrastructure is based on a multi-agent system architecture to integrate various components of the environments. The infrastructure includes a location- and context-awareness service, a question and answer service, an adaptive mechanism; problem based ubiquitous learning models, social networking issues, and the evaluation of multimedia inputs. Furthermore, student modeling issues among components are considered. The design of the infrastructure as well as its components is discussed. This paves the way towards the development of pervasive learning applications.
\end{abstract}

\section{Introduction}

Many of the computer-based applications in elearning focus on either providing tools for the teachers to design courses (e.g., Macromedia Coursebuilder for Dreamweaver, Integrator2, and Toolbook 9) or for learning management (e.g., WebCT, Sakai, and Moodle). These and other existing learner-centered educational applications have limitations in promoting adaptivity and context awareness and providing realtime support to learners from an educational point of view [1].

The usefulness of adaptivity and context awareness is derived from the ability to better support a variety of learners, given that they may have very different skills and motivations to learn in varying contexts. The recent research on adaptivity and context awareness has turned towards supporting pervasive environments, coupled with an increasing trend to examine the learning process from an informal learning perspective [2]. Pervasive learning is a social process that connects learners to communities of devices, people, and situations, including other pervasive leaning situations [3]. In addition to increasing accessibility, mobile devices have the potential to increase connections between students, tutors, and instructors and decrease isolation in distance education [4]. The proliferation of wireless and mobile technologies has increased flexibility for learners and the boundaries of anytime, anywhere learning [5].

Context-aware computing facilitates the use of applications that are aware of contextual information, including time, nearby people and devices, and user activity in a mobile environment [6]. Approaches have been identified for modularizing and contextualizing learning objects [7].

This paper is organized as follows: Section 2 gives an overview about the proposed infrastructure and its components. Section 3 focuses on the integration platform using Multi-Agent system technology. Section 4 discusses the student modeling issues in order to provide relevant data for all components. In the subsequent sections, each component of the infrastructure is described in more detail. The conclusion and future work are presented in Section 12.

\section{Overview}

Figure 1 illustrates the proposed infrastructure for problem-based pervasive learning environments. A fundamental design consideration is to use the multiagent system (MAS) paradigm to seamlessly integrate 
and deploy software components, devices, learners, educational services, and situations to form pervasive learning communities and facilitate collaborative problem solving. Another important design consideration is to have a student model and a student modeling agent which captures specific information about the learner such as learning styles, location, context, behaviors, actions, and performance. The student modeling agent is responsible for gathering the required data from the respective components and making the information accessible for all components. The components themselves are responsible for providing learners with an adaptive and pervasive learning environment. The location-awareness service is used to help mobile students forming face-to-facelearning groups. Moreover, social networking issues are considered and integrated in the infrastructure. An adaptive mechanism is included, which is responsible for providing learners with learning materials that fit to their individual learning styles. The context-awareness service identifies the personalized context-aware knowledge structure in a ubiquitous/pervasive learning environment and directs individual students to learn and move in the real world using automatically generated guidance messages. Furthermore, students are supported by an intelligent and multimodal asynchronous Q\&A knowledge sharing platform. Another component deals with providing effectively integration of problem based learning in adaptive and ubiquitous learning environments. Moreover, different multimedia inputs are considered for providing a richer source of interaction in mobile learning.

The mentioned components can be integrated in a learning environment, providing learners with rich learning experience. This environment supports pervasive and mobile learning as well as problembased learning. Furthermore, it aims at providing personalized and adaptive support for students.

In the following sections, the mentioned design considerations and components of the infrastructure are described in more detail.

\section{The multi-agent architecture}

The term agent is used to refer to an active component which has autonomous, communication, and proactive features [8]. The MLCS (Mobile Learning Communities Service) is a learning support system in complex and open environments. It must employ cooperative software agents to support active and adaptive learning. Each agent focuses on its own specialized tasks, manages its own resources and generates its specialized dynamic view.
JADE-LEAP (Java Agent Development Environment - Lightweight Extensible Agent Platform, http://jade.tilab.com/) was chosen to develop FIPA-compliant MAS in mobile devices because JADE has a run-time for J2ME-CLDC (Connected Limited Device Configuration) and CDC (Connected Device Configuration) platforms.

The MAS-based architecture allows great flexibility and scalability in the integration of components. It provides a simple yet extensible and powerful software layer to develop further pervasive learning environments, while simultaneously running multiple stationary and mobile agents on a CLDC/MIDP equipped resource-limited mobile device [9]. JADELEAP serves as the agent platform.

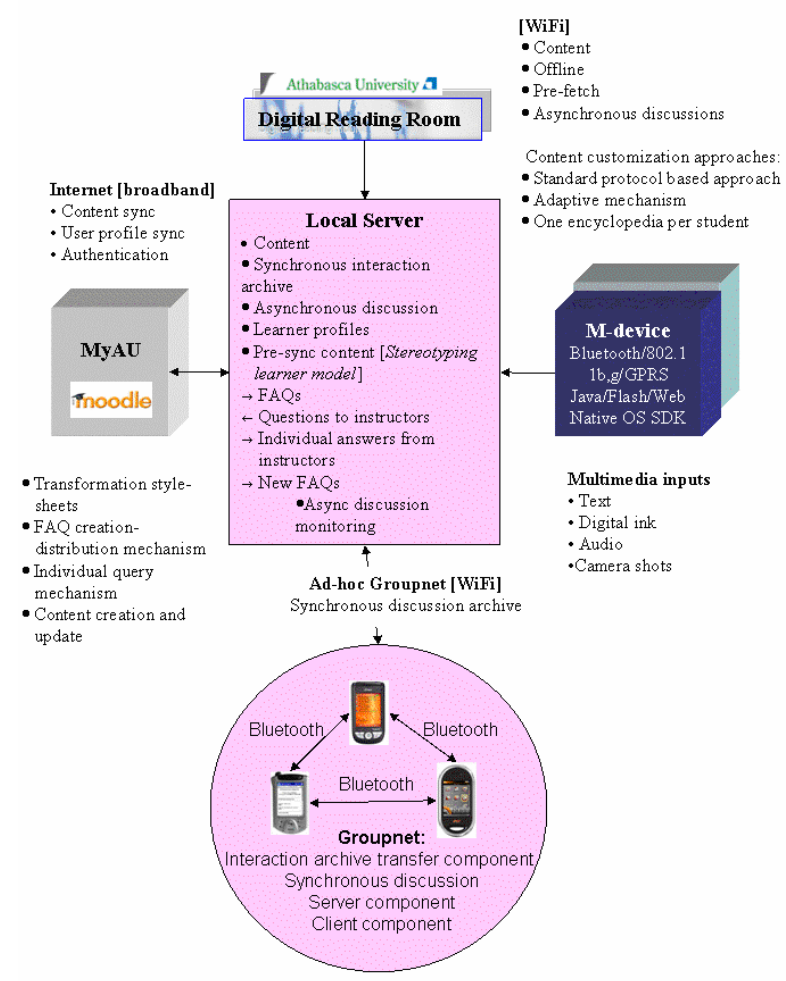

Figure 1. Overview of the proposed infrastructure

Each learner is running the front-end of a learner agent on his/her own mobile phone or PDA supporting J2ME with the Mobile Information Device Profile (MIDP) 1.0 or greater APIs and Bluetooth JSR-82 or Wi-Fi APIs. A Back-end Mediator in the JADE-LEAP architecture, running on a host with a known, fixed, and visible address, can automatically manage the back-ends of all front-ends active on the learner's device. Some types of agents located in a JADE agent platform are as follows: 
- A main container, which stores default JADE agents;

- One or more containers, which execute system agents;

- Location-aware learner agents, one for each learner, which present each learner logged in within the area. The learner agent has capabilities, using the expressed preferences of learners or their previous behavior when navigating the system to provide information for the student model.

- Connection agents manage the connection between the mobile devices and agent platform.

- Service agents are available for each service such as location-aware service.

- Resource agents can manage resources, such as problem-based learning objects.

Figure 2 shows the MAS-based system architecture for pervasive learning.

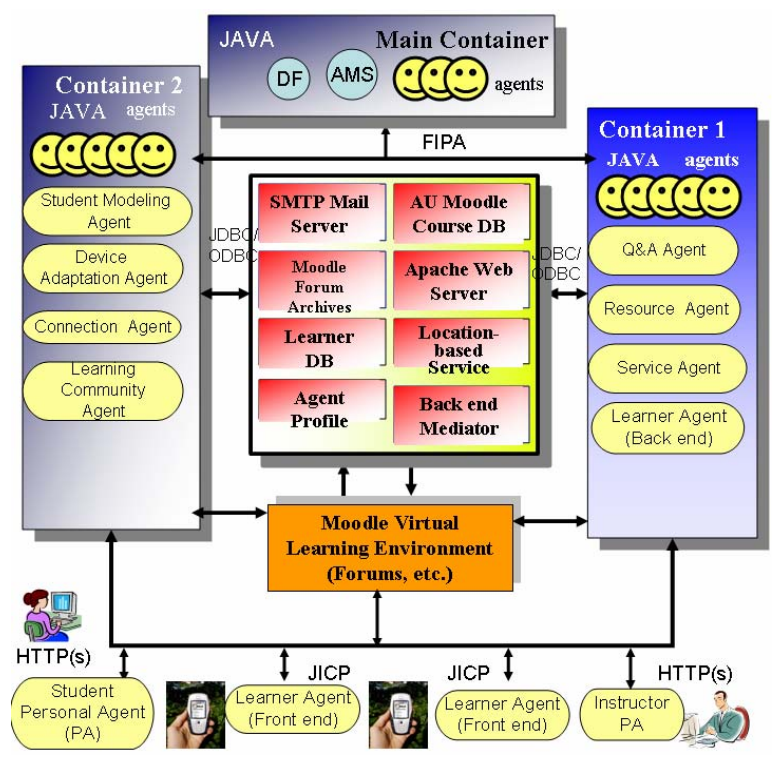

Figure 2: The system architecture.

\section{Student Modeling}

When aiming at providing learners with an environment that adapts to their individual characteristics, interests and needs, this information about learners has to be discerned by the system. A student model is a crucial component of every adaptive system. It stores and updates the relevant information about learners which is needed by the system in order to provide adaptivity [10]. In the proposed infrastructure, we are building a global student model that links different components together. This global student model is being built on the multi-agent architecture-based platform described in the previous section, and cooperates with all other components in order to gather relevant data from the components and make them accessible to all the other components.

Such a global student model has several benefits. First, data which are used by more than one component need to be gathered only once and can then be used by all other components. On the other hand, when data need to be gathered frequently in order to update the student model, data can be gathered from different components, which leads to more information in order to build a more accurate student model. Furthermore, data which are needed by a certain component, might be easily gathered by other components and can therefore be provided in the global student model.

However, the components themselves should not be dependent on each other. Accessing information provided from another component should be an option, but if this information is not available the component has to be able to detect the required information by itself and then provide the information in the global student model so that other components can access and use it.

\section{Location-awareness service}

This service provides a location based optimal grouping algorithm which is designed to group the individual mobile learners into face-to-face learning groups based on the identification of their geographic locations, learning behaviors, and other features. Applying location awareness in pervasive learning provides another new dimension for mobile learners, which enables them to take advantage of collaborative learning, and can enhance the students' learning in the pervasive learning environment.

A location awareness service based on the optimal grouping algorithm is being implemented in the MASbased system architecture. A location-aware learner agent (front-end) running on a mobile device will periodically acquire available location information from GPS satellites, or base stations of cellular networks, or access points for Wi-Fi broadband wireless networks. The acquired location data will be preprocessed and then fed to the location awareness server as location input. On the server side, a locationaware learner agent running in a JADE agent platform will use the location input combining with other available data to propose an optimal online grouping option for the mobile students.

The location based optimal grouping algorithm includes the detection of the location information availability, the adaptation to various mobile devices in 
the market, the acquisition and preprocessing of the location data on the mobile device, processing of the location data on a location awareness service server, incorporation of the location data and other learning factors, and the appropriate grouping of mobile students.

\section{Social networks issues}

It is anticipated that a significant number of students will already be participating in online social networks outside the institution. A recent study suggests that up to $96 \%$ of young online users engage in social sites such as MySpace, Facebook, YouTube and Flickr [11].

One of the most significant issues when exchanging data between informal social networks and the more structured groups of formal education is control. On the one hand, not all students are willing to have their private life information accessed by their educational institutions [12]. On the other hand, the danger of spamming, malware or malicious and mischievous outsiders invading the trusted space of educational institutions is real. Institutional Web sites are open and potentially abusable by students, leading to legitimate criticism of the wild and uncontrollable Web 2.0 [13]. An interesting research challenges is to provide a controllable, safe interaction between these formal and informal worlds. Our agents need to be able to ascertain and assert trust at both system and personal levels to help bridge the trust and credibility gaps that are becoming an increasing issue on the social Web [13].

The components rely on technologies outside the system. In some instances, standards such as FOAF and RSS may be used to gather data. Existing standards such as Shibboleth, LDAP and OpenID are brought into play to help address issues of trust automatically. However, the tempestuous and commercially driven world of social software does not always adhere to standards and sometimes goes out of its way to maintain insularity. Unfortunately, some of the largest sites (e.g., MySpace, Facebook) are among the worst offenders in this respect. In such cases, we have to construct components, widgets or mashups built using tools specific to social sites to interface with our agents. This in itself raises concerns about reliability, continuity and trust. Our agents need to be able to cope with sites that may change, disappear, or fall victim to malicious users, degrading gracefully and handing over functionality where necessary to different systems.

\section{Adaptive mechanism}

The adaptive mechanism focuses on the consideration of learning styles based on the FelderSilverman learning style model [14]. In order to provide learners with courses that fit to their individual learning styles, we aim at developing an extension for the learning management system Moodle. The adaptive mechanism is designed in a generic way, giving teachers and administrators the possibility to add whatever kind of learning object they want to use in their adaptive courses. As a result, teachers can continue using the LMS, while taking full advantage of its enhanced features. Additionally, the adaptive mechanism facilitates the provision of courses that more closely fit learners' different learning styles and therefore promote learning.

\section{Content-awareness service}

The context-awareness service is built on the multiagent architecture-based platform and cooperates with other agents. The service aims at taking individual's interest(s) and/or request(s) from a student modeling agent and a learning community agent to identify a specific context-aware knowledge structure among the knowledge structures in different domains in a pervasive/ubiquitous learning environment [15]. Once the personalized context-aware knowledge structure has been found and reorganized, the system can lead individual learners in the real world facilitating selfstudy and/or social networking with others for group learning [16- 18].

In order to help a user plan his/her learning activities when learning in the real world, an ubiquitous/pervasive learning system should be able to know what the user is looking for and/or really interested in $[19,20]$. Furthermore, to give the user the appropriate learning guidance, the learning system has to know where the user is and what related learning objects are present in the environment. This research uses the context-awareness knowledge structures to represent the learning environment; to identify the learning objective(s) that the user is really interested in; to propose learning activities to the user; and, to lead the user around the learning environment.

\section{Question \& answer service}

As a direct and convenient way to exchange and share knowledge, question/answer support is important in problem-based learning. Presented as Frequently Asked Questions (FAQs), this type of support is quite common and appears as an essential part of many 
online learning environments. This component promotes the current Question \& Answer (Q\&A) services such as discussion forums and adaptive FAQ to an integrated system by providing common Q\&A knowledge representation and deeper questions and answers processing through question answering (QA) technology. Despite the different forms of interfaces of FAQ or discussion forums, the internal mechanisms and representations of them are very similar, i.e. all associating with questions and relevant answers to these questions. This encourages us to combine them together. QA [21] is based on natural language processing (NLP) and information retrieval (IR) and aims to automatically retrieve answers from specific resources to students' questions in natural language text. It provides techniques related to analyze questions, retrieve and generate answers from natural language text resources. Figure 3 shows the overall structure of this component. An FAQ authoring tool provides a convenient function for picking up questionanswer pairs directly from discussion forums, and question analysis and automatic answer extraction helps to get potential Q\&A pairs from the threads of discussion forums and from domain ontologies by adopting ontology querying and reasoning. An important feature of this component is the use of context information from the context-aware service and student profiles. The component is designed to be compatible with Moodle discussion forums, eQuake [22], and is based on open source tools such as Lucene, Opennlp and Gate.

The main functions of this component include discussion forums with some value-added functions inherited from eQuake such as answer type feedback, forum monitoring and notification [23], FAQ authoring, deeper understanding of questions and effective answering by QA, and mobile learning environment support.

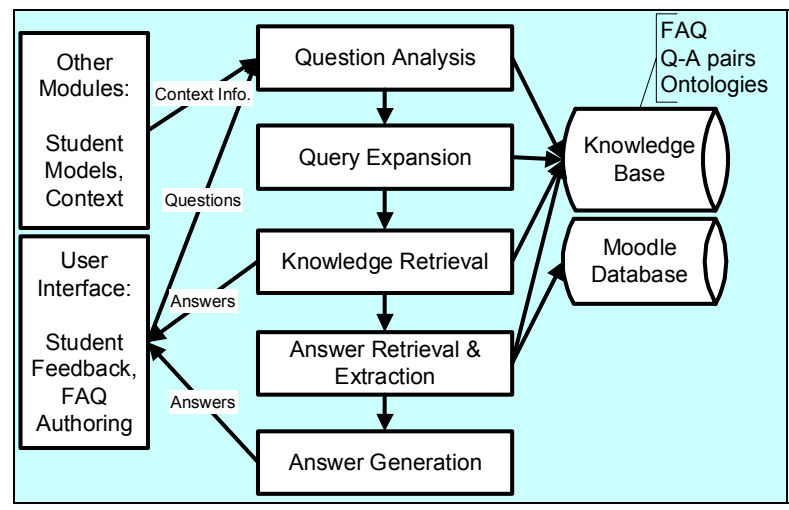

Figure 3. Q \& A service

\section{Problem-based ubiquitous learning models}

Problem-based learning $(\mathrm{PBL})$ is a learner-centered approach. It is grounded in cognitive theories and focuses on putting learners in real-word problems to enhance their learning motivation, skills of problem solving and knowledge construction. When implementing problem-based learning, learners should have rich interactions with content, contexts, and other learners. Ubiquitous learning environments have the potential to provide multiple channels to support different interactions (such as face-to-face and computer mediated communication) anytime and anywhere. Moreover, adaptive learning environments can help students learn at their own pace and as per their individual preferences. Therefore, integrating PBL, adaptive and ubiquitous learning environments have the potential to significantly improve and enhance learning.

However, as with any learning methods, there are many learning models for implementing PBL depending on the learning goals and the features of learning environments. Both ubiquitous and adaptive learning environments are new areas of research. Few researchers have investigated how these environments can fit the needs of PBL and how to implement PBL in these environments. Previous investigations, such as [24], have suggested that applying innovative technology inappropriately can generate negative effectiveness. In this study, we aim at designing, developing, introducing, implementing, and evaluating learning models that integrate PBL in adaptive learning environments and ubiquitous learning environments effectively.

\section{Multimedia input}

Use of multimedia enables rich environment for learning. This component of the system focuses on using the rich input facilities, such as text, images, voice and digital ink, offered by mobile devices to enhance interaction. Our research centers on how multimedia based learning environments promote constructive learning which fosters problem-solving skills. On a mobile device, learners have access to text (via keyboard and digital ink), images (via the camera), and sound (via the microphone) on a relatively small, portable device. The main emphasis behind our work is to develop a concept for the effective use of multimedia input in mobile devices in order to support learning by offering a rich source of interaction. 


\section{Conclusions and future work}

In this paper, we have proposed a multi-agent system based architecture to support adaptive and pervasive learning. The set of specific agents, models, services and adaptive mechanism needed are described. This set builds a rich infrastructure for pervasive learning, where each component contributes for supporting mobile students to learn by considering their individual needs and providing them with an adaptive environment. Further work deals with completing implementation issues. Therefore, the infrastructure will be combined with the learning management system Moodle and evaluated in order to show the effectiveness of the proposed infrastructure.

\section{References}

[1] M. C. Rosatelli and J. A. Self, A Collaborative Case Study System for Distance Learning, International Journal of Artificial Intelligence in Education, 14, 2004, pp. 1-29.

[2] A. Syvanen, R. Beale, M. Sharples, M. Ahonen, and P. Lonsdale, Supporting Pervasive Learning Environments: Adaptability and Context Awareness in Mobile Learning, Proceedings of the International Workshop on Wireless and Mobile Technologies in Education, 2005, pp.251-253.

[3] S. Thomas, Pervasive, Persuasive eLearning: Modeling the Pervasive Learning Space, Proceedings of the Pervasive Computing and Communications Workshops, 2005, pp. 332-336

[4] T. Hulsmann, The Two-Pronged Attack on Learner Support: Costs and Centrifugal Forces of Convergence. Proceedings of the Third EDEN Research Workshop (electronic version), 2004. Retrieved March 31, 2005.

[5] R. McGreal, Mobile Technologies and the Future of Global Education, Proceedings of ICDE International Conference on Open Learning and Distance Education, New Delhi, India, Nov. 19-23, 2005.

[6] G. Chen and D. Kotz, A Survey of Context-Aware Mobile Computing Research, Dartmouth Computer Science Technical Report, TR2000-381, 2000.

[7] A. Schmidt and C. Winterhalter, User Context Aware Delivery of E-Learning Material: Approach and Architecture, Journal of Universal Knowledge Management, 10(1), 2004, pp. 28-36.

[8] M. Wooldridge, N. Jennings; (1995); Intelligent agents: Theory and practice; The Knowledge Engineering Review; 10(2); 115 - 152; Cambridge University Press.

[9] F. Bellifemine, G. Caire, \& D. Greenwood, Developing Multi-Agent Systems with JADE, John Wiley \& Sons, Ltd, 2007.

[10] P. Brusilovsky, The construction and application of student models in intelligent tutoring systems, International Journal of Computer and Systems Sciences, 32, 1994, pp. 70-89.
[11] Grunwald Associates LLC, Creating \& Connecting: Research and Guidelines on Online Social and Educational Networking, National School Boards Association 2007.

[12] A. Keen, The Cult of the Amateur. Nicholas Brealey Publishing, London, 2007.

[13] J. N. Hoover, Web Credibility: Hard Earned, Harder To Prove, Information Week, June 4, 2007.

[14] R. M. Felder and L. K. Silverman, Learning and Teaching Styles in Engineering Education. Engineering Education, 78(7), 1988, pp. 674-681.

[15] A. Chang, K.-F. Cheng, M. Chang and J.-S. Heh, Measuring the Consistency of Concept Abstraction and Similarity of Concept Map from Discussion Database in the PBL Learning Environment. Proceedings of the Annual Global Chinese Conference on Computers in Education, Hawaii, USA, June 6-9, 2005, pp. 677-685.

[16] M. J. Egenhofer and R. D. Franzosa, On the Equivalence of Topological Relations. International Journal of Geographical Information Systems, 9(2), 1995, pp. 133152.

[17] M. E. Sorrows \& S. C. Hirtle, The Nature of Landmarks for Real and Electronic Spaces. Proceedings of the International Conference on Spatial Information Theory: Cognitive and Computational Foundations of Geographic Information Science, Stade, Germany, August 25-29, 1999. LNCS, vol. 1661, Berlin: Springer Verlag, 1999, 37-50.

[18] B. Tversky \& P. Lee, Pictorial and Verbal Tools for Conveying Routes, Proceedings of the International Conference on Spatial Information Theory: Cognitive and Computational Foundations of Geographic Information Science, LNCS, vol. 1661, Springer Verlag, Berlin, 1999, 51-64.

[19] H. Ogata \& Y. Yano. Context-Aware Support for Computer-Supported Ubiquitous Learning. Proceedings of the 2nd IEEE International Workshop on Wireless and Mobile Technologies in Education, Jhongli, Taiwan, March 23-25, 2004, pp. 27-34.

[20] A. Chang \& M. Chang, Adaptive Mobile Navigation Paths for Elementary School Students' Remedial Studying. Proceedings of the IEEE International Conference on Interactive Computer Aided Learning, Villach, Austria, Sept. 27-29, 2006.

[21]Z. Chen \& D. Wen, Accelerating and Evaluation of Syntactic Parsing in Natural Language Question Answering Systems. Proceedings of International Conference on Artificial Intelligence, ICAI'07, June 2528, Las Vegas, Nevada, USA, 2007.

[22] eQuake, 2006, Available at http://www.equake.org.nz/.

[23] J. Yang, Ø. Smestad, Kinshuk, Y. Zhang, L. Jeffrey, \& A. Tretiakov, Electronic Question \& Answer Knowledge Environment Forum. Proceedings of the Int. Conf. on Computers in Education, Beijing, China, Nov. 20-24, 2006.

[24] T. C. Liu, Teaching in a wireless learning environment: a case study. Educational Technology \& Society, 10(1), 2007, pp. 107-123. 